\title{
GEODESICALLY TRACKING QUASI-GEODESIC PATHS FOR COXETER GROUPS
}

\author{
MICHAEL MIHALIK AND STEVE TSCHANTZ
}

\begin{abstract}
If $\Lambda$ is the Cayley graph of a Gromov hyperbolic group, then it is a fundamental fact that quasi-geodesics in $\Lambda$ are tracked by geodesics. Let $(W, S)$ be a finitely generated Coxeter system and $\Lambda(W, S)$ the Cayley graph of $(W, S)$. For general Coxeter groups, not all quasi-geodesic rays in $\Lambda$ are tracked by geodesics. In this paper we classify the $\Lambda$-quasi-geodesic rays that are tracked by geodesics. As corollaries we show that if $W$ acts geometrically on a $\operatorname{CAT}(0)$ space $X$, then CAT(0) geodesics in $X$ are tracked by Cayley graph geodesics (where the Cayley graph is equivariantly placed in $X$ ) and for any $A \subset S$, the special subgroup $\langle A\rangle$ is quasi-convex in $X$. We also show that if $g$ is an element of infinite order for $(W, S)$ then the subgroup $\langle g\rangle$ is tracked by a Cayley geodesic in $\Lambda(W, S)$ (in analogy with the corresponding result for word hyperbolic groups).
\end{abstract}

\section{INTRODUCTION}

Suppose $G$ is a group with finite generating set $A$, and $\Lambda(G, A)$ is the Cayley graph of $G$ with respect to $A$. If $G$ is word hyperbolic then any quasigeodesic in $\Lambda$ is tracked by a geodesic (see [Sh]). The corresponding result for CAT(0) groups is not true. Our main goal in this paper is to classify the quasi-geodesics in the Cayley graph of a finitely generated Coxeter system that are tracked by geodesics. We define a "bracket number" for a Cayley path in terms of the wall crossings of the path and our main theorem is that a quasi-geodesic ray or line is tracked by a geodesic iff the bracket number of the ray (line) is bounded. Our principal corollary to this theorem states that if $(W, S)$ is a finitely generated Coxeter system, and $W$ acts geometrically on a $\operatorname{CAT}(0)$ space $X$, then the $\mathrm{CAT}(0)$ geodesics of $X$ are tracked by $(W, S)$ Cayley geodesics in $X$. If $X$ is the Davis complex for $(W, S)$ or even if $W$ acts as a reflection group on $X$, the proof of the corollary is straightforward. Unfortunately, the reflection group argument has no analogue when $W$ does not act as a reflection group on $X$. The principal corollary directly implies that if $A \subset S$ then the special subgroup $\langle A\rangle$ is quasi-convex in $X$.

If a group $G$ acts geometrically on a $\mathrm{CAT}(0)$ space $X$ and one is interested in the asymptotic properties of $X$ it is a considerable advantage to know that $\mathrm{CAT}(0)$ geodesics in $X$ are tracked by Cayley geodesics. Clearly,

Date: October 30, 2018.

Key words and phrases. CAT(0) group, Coxeter group, quasi-geodesic. 
the algebraic properties of $G$ are far more apparent in Cayley geodesics than in CAT(0) geodesics. This theme is highlighted in [MRT] where local connectivity of boundaries of right angled Coxeter groups are analyzed.

The work of B. Bowditch and G. Swarup (see $[\mathbf{S}]$ ) imply that 1-ended word hyperbolic groups have locally connected boundary. One can easily see from our tracking results that any 1-ended hyperbolic Coxeter group has locally connected boundary.

\section{Coxeter Preliminaries}

We use M. Davis' book D] as a general Coxeter group reference for this section. A Coxeter system is a pair $(W, S)$ where $S$ is a generating set for the group $W$ and $W$ has presentation

$$
\left\langle S:\left(s_{i} s_{j}\right)^{m(i, j)} \text { for all } s_{i}, s_{j} \in S\right\rangle
$$

where $m(i, j) \in\{1,2, \ldots, \infty\}, m(i, j)=1$ iff $i=j$ (so all generators are order 2) and $m(i, j)=m(j, i)$. If $m(i, j)=\infty$, the element $s_{i} s_{j}$ is of infinite order (and the relation $\left(s_{i} s_{j}\right)^{\infty}$ is left out of the presentation).

A reflection in $W$ is a conjugate of an element of $S$. If $w \in W$ and $s \in S$ then the edge labeled $s$ in the Cayley graph $\Lambda(W, S)$ at the vertex $w$ is mapped to itself by the reflection $w s w^{-1}$, so that the vertices $w$ and $w s$ are interchanged. I.e. the edge is reflected across its midpoint. The set of edges in $\Lambda$ each fixed (set-wise) by a reflection is a wall of $\Lambda$. The walls of $\Lambda$ partition the edges of $\Lambda$ into disjoint sets. Notationally, we write a wall $Q$ as $[e]$ where $e$ is any edge of the wall $Q$ and we define $\bar{Q}$ to be the union of the edges of $Q$ in $\Lambda$. An edge $e$ (with say label $t \in S$ ) belongs to a wall $Q$ corresponding to the reflection $w s w^{-1}$ iff a vertex of $e$ is $w q$ where $q t q^{-1}=s$. The closure of the compliment of a wall in $\Lambda$ has exactly two components (which are interchanged by the reflection) called the sides of the wall. Two walls are parallel if all edges of one are on the same side of the other. If two walls are not parallel, then they cross. The following theorem due to B. Brink and R. Howlett (see theorem 2.8 of $[\mathrm{BrH}]$ ) is a fundamental result concerning the wall structure of $\Lambda$.

Theorem 2.1. (Parallel Wall theorem) Suppose $(W, S)$ is a finitely generated Coxeter system and $\Lambda(W, S)$ the Cayley graph of $W$ with respect to $S$. For each positive integer $n$ there is a constant $P(n)$ such that the following holds: given a wall $Q$ and a point $p$ in $\Lambda$ such that the distance from $p$ to $\bar{Q}$ is at least $P(n)$, then there exist $n$ distinct pairwise parallel walls which separate $\bar{Q}$ from $p$.

For a path $\beta$ in $\Lambda$ and vertex $t$ of $\beta$ let the bracket number of $t$ in $\beta$ be the number of walls $Q$ such that there is an edge of $Q$ on either side of $t$ in $\beta$. Denote the bracket number of $t$ in $\beta$ as $B(t, \beta)$. If $\tau$ is a subpath of $\beta$ the bracket number of $\tau$ in $\beta$ is the maximum of the numbers $B(t, \beta)$ for all vertices $t$ of $\tau$. Denote this number $B(\tau, \beta)$. Call $B(\beta) \equiv B(\beta, \beta)$ the bracket number of $\beta$. 


\section{WALl COMPUTATIONS}

If $\alpha$ is an edge path in the Cayley graph $\Lambda$ with consecutive vertices $a=v_{0}, v_{1}, \ldots, v_{n}=b$, then an $L$-approximation to $\alpha$ is an edge path in $\Lambda$ connecting $a$ and $b$ of the form $\left(\alpha_{1}, \ldots, \alpha_{n}\right)$ where for all $i, \alpha_{i}$ is geodesic connecting $w_{i-1}$ to $w_{i}$ and $w_{i}$ is within $L$ of $v_{i}$. The points $w_{i}$ are called approximation points.

Lemma 3.1. Suppose $(W, S)$ is a finitely generated Coxeter system, $\alpha$ is an edge path in the Cayley graph $\Lambda(W, S)$ connecting $a$ and $b$, and $\beta$ is an $L$ approximation of $\alpha$. Then the bracket number $B(\beta)$ is bounded by a constant only depending on $B(\alpha), L$ and constants independent of the choice of $\alpha$.

Proof. Let the consecutive vertices of $\alpha$ be $a=v_{0}, v_{1}, \ldots, v_{n}=b$, the approximation vertices of $\beta$ be $a=w_{0}, w_{1}, \ldots, w_{m}=b$ (so that $d_{\Lambda}\left(w_{i}, v_{i}\right) \leq L$ for all $i$ ) and $\beta_{i}$ be the geodesic subpath of $\beta$ connecting $w_{i-1}$ to $w_{i}$. Then $\beta=\left(\beta_{1}, \ldots, \beta_{m}\right)$. If $x$ is a vertex of $\beta_{i}$ and $B(x, \beta)$ is "large", then (as each edge belongs to exactly one wall) there is a wall $Q$ that brackets $x$ on $\beta$ that is "far" from $x$ and hence far from $v_{i}$. Hence it suffices to bound the distance between $v_{i}$ and a wall $Q$ that brackets $x$ on $\beta$. The Parallel Wall theorem implies this distance is large iff there is a large set $\mathcal{Q}$ of (mutually parallel) walls that separate $\bar{Q}$ from $v_{i}$, so it suffices to bound the size of the set $\mathcal{Q}$ of walls that separate $\bar{Q}$ from $x$. Say $j<i<k$ such that $e_{j}$ and $e_{k}$ are edges of $\beta_{j}$ and $\beta_{k}$ respectively, and each of $e_{j}, e_{k}$ belongs to the wall $Q$. (See figure 1)

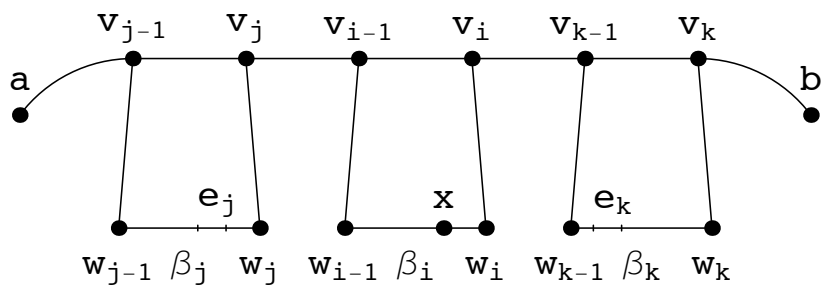

Figure 1

A path $\delta_{j}$, that begins at the end point of $e_{j}$ follows $\beta_{j}$ to $w_{j}$ and then travels geodesically from $w_{j}$ to $v_{j}$ has length $\leq 3 L$. If $\alpha_{j, i}$ is the subpath of $\alpha$ from $v_{j}$ to $v_{i}$, then the path $\left(\delta_{j}, \alpha_{j, i}\right)$ must cross each wall of $\mathcal{Q}$. Similarly define a path from $e_{k}$ to $v_{i}$ (which also crosses each wall of $\mathcal{Q}$ ). Then at most $6 L$ walls of $\mathcal{Q}$ do not bracket $v_{i}$ on $\alpha$. This bounds the size of $\mathcal{Q}$ by $6 L+B(\alpha)$.

Lemma 3.2. Suppose $(W, S)$ is a Coxeter system and $\alpha=\left(e_{1}, \ldots, e_{n}\right)$ is a geodesic edge path connecting vertices $a$ and $b$ in $\Lambda(W, S)$ such that $\alpha$ does not cross the wall $Q$. If $e_{0}$ is an edge at $a$ and $e_{n+1}$ an edge at $b$ such that $e_{0}$ and $e_{n+1}$ belong to the wall $Q$ then each vertex of $\alpha$ is within $P(1)$ of $\bar{Q}$ (where $P$ is the function of theorem 2.1). In particular, if $v$ is a vertex of $\alpha$ and $v^{\prime}$ the reflection of $v$ across $Q$ then $d\left(v, v^{\prime}\right) \leq 2 P(1)+1$. 
Proof. Otherwise, there is a wall $Q^{\prime}$ separating a vertex $v$ of $\alpha$ from $Q$. Hence there is an edge of $\alpha$ between $a$ and $v$ that belongs to $Q^{\prime}$ and an edge of $\alpha$ between $v$ and $b$ that belongs to $Q^{\prime}$. This is impossible as $\alpha$ is geodesic.

Proposition 3.3. Suppose $(W, S)$ is a Coxeter system and $\alpha$ is an edge path of $\Lambda(W, S)$ connecting $a$ and $b$. Then there is an L-approximation $\beta$ to $\alpha$ such that each vertex of $\beta$ is on a geodesic connecting $a$ and $b$ and such that $L \leq(2 P(1)+1) B(\alpha)$.

Proof. Let the consecutive vertices of $\alpha$ be $a=v_{0}, \ldots, v_{n}=b$. For $0<i<n$ we choose an approximation point $w_{i}$ for $v_{i}$ as follows. Let $\alpha_{i}$ be the geodesic from $a$ to $v_{i}$ and $\beta_{i}$ the geodesic from $v_{i}$ to $b$. Each wall of $\left(\alpha_{i}, \beta_{i}\right)$ is crossed exactly once or twice. The number of walls crossed twice by $\left(\alpha_{i}, \beta_{i}\right)$ is

$$
N_{i} \equiv \frac{1}{2}\left(d\left(a, v_{i}\right)+d\left(v_{i}, b\right)-d(a, b)\right) \leq B(\alpha)
$$

Let $e$ be the last edge of $\alpha_{i}$ belonging to a wall which is crossed twice by $\left(\alpha_{i}, \beta_{i}\right)$ and $d$ the edge of $\beta_{i}$ in the same wall as $e$. (See figure 2.)

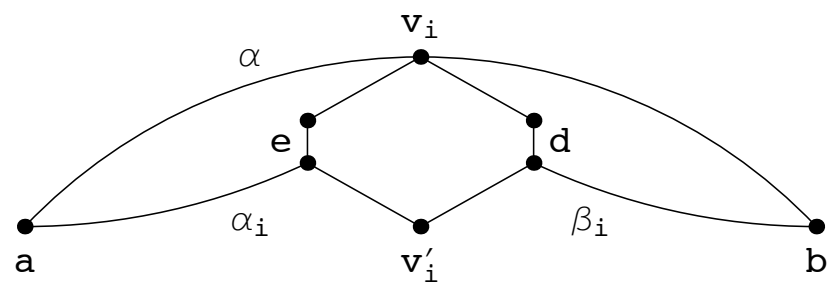

Figure 2

The segment of $\left(\alpha_{i}, \beta_{i}\right)$ between $e$ and $d$ is geodesic. Considering the reflection of this segment across the wall containing $e$ and $d$ (equivalently, delete $e$ and $d$ from $\left.\left(\alpha_{i}, \beta_{i}\right)\right)$. Then we see that $v_{i}^{\prime}$, the reflection of $v_{i}$, is within $2 P(1)+1$ of $v_{i}$ (lemma 3.2 ), and the distance from $v_{i}^{\prime}$ to $a$ (respectively $b)$ is less than that of $v_{i}$ to a (respectively $\left.b\right)$. Hence $\frac{1}{2}\left(d\left(a, v_{i}^{\prime}\right)+d\left(v_{i}^{\prime}, b\right)-\right.$ $d(a, b))<N_{i}$ and a geodesic from $a$ to $v_{i}^{\prime}$ followed by a geodesic from $v_{i}^{\prime}$ to $b$ crosses at most $N_{i}-1$ walls twice. Continuing as above at most $N_{i}(\leq B(\alpha))$ such reflections are needed to move $v_{i}$ to a point $w_{i}$ on a geodesic between $a$ and $b$, and so $d\left(w_{i}, v_{i}\right) \leq(2 P(1)+1) B(\alpha)$.

It remains to see that each vertex of a geodesic connecting $w_{i}$ and $w_{i+1}$ belongs to a geodesic connecting $a$ and $b$. Consider the edge path $\left(\delta_{i}, \beta_{i}, \gamma_{i}\right)$ where $\delta_{i}$ is a geodesic connecting $a$ to $w_{i}, \beta_{i}$ is a geodesic connecting $w_{i}$ to $w_{i+1}$ and $\gamma_{i}$ is a geodesic connecting $w_{i+1}$ to $b$. The paths $\delta_{i}$ and $\gamma_{i}$ only cross walls crossed by some (equivalently any) geodesic connecting $a$ to $b$. If a vertex $v$ of $\beta_{i}$ is not on a geodesic connecting $a$ and $b$ then there is a wall $R$ separating $v$ from some (equivalently every) geodesic connecting $a$ and $b$. As $R$ separates $v$ from $a$, and $\delta_{i}$ does not cross $R, \beta_{i}$ must cross $R$ between 
$w_{i}$ and $v$. Similarly $\beta_{i}$ must cross $R$ between $v$ and $w_{i+1}$. This is impossible as $\beta_{i}$ is geodesic.

If $\gamma$ is an edge path in $\Lambda$ connecting the vertices $a$ and $b$, then each wall separating $a$ and $b$ is crossed an odd number of times by $\gamma$ and each wall not separating $a$ and $b$ is crossed and even number of times by $\gamma$. If two edges of $\gamma$ belong to the same wall then they may be "deleted" to obtain another path from $a$ to $b$ (i.e. if edges $e$ and $d$ of $\gamma$ belong to the same wall $Q$, and $\tau$ is the segment of $\gamma$ between $e$ and $d$, then $(e, \tau, d)$ can be replaced in $\gamma$ by $\tau^{\prime}$, where $\tau^{\prime}$ is the reflection of $\tau$ across $Q$, to obtain a shorter path connecting $a$ and $b$ ). If $\alpha$ is a geodesic connecting $a$ and $b$ then the walls separating $a$ and $b$ are the walls determined by the edges of $\alpha$, so the walls separating $a$ and $b$ are in 1-1 correspondence with the edges of some (any) geodesic connecting $a$ and $b$. The following observations are straightforward.

Lemma 3.4. Suppose $\beta$ is an edge path in $\Lambda$ connecting the vertices a and $b$ such that each vertex of $\beta$ is on a geodesic connecting $a$ and $b$. Then

i) each edge of $\beta$ belongs to a wall that separates a from $b$,

ii) each wall crossed by $\beta$ is crossed an odd number of times, and

iii) if $c$ and $d$ are vertices of $\beta$ then any wall separating $c$ and $d$ also separates $a$ and $b$.

The next result is a slightly more sophisticated version of lemma 3.2 .

Lemma 3.5. Suppose $\alpha$ is a geodesic edge path in $\Lambda$ connecting the vertices $a$ and $b, v$ is a vertex of $\alpha$, and $a$ and $b$ are each within distance $A$ of $\bar{Q}$ for some wall $Q$. Then $v$ is within distance $2 A(2 P(1)+1)+P(1)$ of $\bar{Q}$.

Proof. Let $a^{\prime}$ (respectively $b^{\prime}$ ) be a vertex of $\bar{Q}$ within $A$ of $a$ (respectively $b$ ) and on the same side of $Q$ as is $a$ (respectively $b$ ). Let $\beta$ (respectively $\gamma$ ) be a geodesic from $a^{\prime}$ to $a$ (respectively $b$ to $\left.b^{\prime}\right)$.

Case 1. The geodesic $\alpha$ does not cross $Q$.

In this case the path $\delta_{0} \equiv(\beta, \alpha, \gamma)$ does not cross $Q$. Since $|\beta| \leq A$ and $|\gamma| \leq$ $A$, a sequence of at most $2 A$ deletions (the first in the path $\delta_{0}$ ) determines a geodesic connecting $a^{\prime}$ to $b^{\prime}$ which does not cross $Q$.

*) Each deletion is taken so that if $e$ and $d$ are the deleted edges, then the subpath determined by $e$ (or $d$ ) along with the subpath between $e$ and $d$ is geodesic.

If $e_{1}$ and $d_{1}$ are the first such deletion edges (so $e_{1}$ and $d_{1}$ are edges of $\delta_{0}$ ) then let $\delta_{1}$ be obtained from $\delta_{0}$ by deleting $e_{1}$ and $d_{1}$. If $v$ is not between $e_{1}$ and $d_{1}$ then $v$ is a vertex of $\delta_{0}$. If $v$ is between $e_{1}$ and $d_{1}$, then $v_{1}$, the reflection of $v$ across the wall $\left[e_{1}\right]=\left[d_{1}\right]$, is within $2 P(1)+1$ of $v$, by lemma 3.2. (Note that the hypotheses of lemma 3.2 are satisfied since we require condition $*$.) In any case $\delta_{1}$ contains a vertex $v_{1}$ within $2 P(1)+1$ of $v$. If $e_{2}$ and $d_{2}$ are deleting edges of $\delta_{1}$ (satisfying $*$ ), then let $\delta_{2}$ by obtained from $\delta_{1}$ by deleting $e_{2}$ and $d_{2}$. Lemma 3.2 implies $\delta_{2}$ contains a vertex $v_{2}$ within 
$2 P(1)+1$ of $v_{1}$ and so within $2(2 P(1)+1)$ of $v$. Inductively, after $K \leq 2 A$ deletions, we obtain a geodesic $\delta_{K}$ connecting $a^{\prime}$ and $b^{\prime}$, and $\delta_{K}$ contains a vertex $v_{K}$ within $K(2 P(1)+1)$ of $v$. Note that $\delta_{k}$ does not cross $Q$. By lemma 3.2, $v_{K}$ is within $P(1)$ of $\bar{Q}$ so that $v$ is within $2 A(2 P(1)+1)+P(1)$ of $\bar{Q}$. This completes case 1 .

Case 2. Suppose $\alpha$ crosses $Q$.

Say the edge $e$ of $\alpha$ between $v$ and $b$ belongs to $Q$. Repeat the case 1 argument with $\delta_{0}$ replaced by $\left(\beta, \alpha^{\prime}\right)$, where $\alpha^{\prime}$ is the subsegment of $\alpha$ from $a$ to the initial point of $e$. Similarly if $e \in Q$ is an edge of $\alpha$ between $a$ and $v$. Note that in both case 2 scenarios, at most $A$ deletions are required to straighten to a geodesic, so the bound is reduced to $A(2(P(1)+1)+P(1)$.

\section{Tracking Quasi-geodesics}

We are interested in quasi-geodesic edge paths in $\Lambda$. An edge path in $\Lambda$ is a continuous map $\beta:[0, n] \rightarrow \Lambda$ such that $n \in \mathbb{Z}^{+}$and for each nonnegative integer $k, \beta$ maps the interval $[k, k+1]$ isometrically to an edge of $\Lambda$. Similarly if $\beta:[0, \infty) \rightarrow \Lambda$, then $\beta$ is called a ray and, if $\beta:(-\infty, \infty) \rightarrow \Lambda$ then $\beta$ is called a line. An edge path $\beta$ is a $(\lambda, \epsilon)$-quasi-geodesic if for each pair of integers $s$ and $t,|s-t| \leq \lambda d(\beta(s), \beta(t))+\epsilon$. If $\alpha$ and $\beta$ are edge paths, then $\beta$ is $K$-tracked by $\alpha$ if each vertex of $\beta$ is within $K$ of a vertex of $\alpha$.

Lemma 4.1. For $i \in\{1,2\}$ suppose $\beta_{i}$ is a $\left(\lambda_{i}, \epsilon_{i}\right)$-quasi-geodesic edge path in $\Lambda, \beta_{1}$ is $K$-tracked by $\beta_{2}$ and $\beta_{1}(0)$ is within $K$ of $\beta_{2}(0)$. Assume both $\beta_{1}$ and $\beta_{2}$ are bi-infinite, or both are rays, or both are finite length and the terminal points of $\beta_{1}$ and $\beta_{2}$ are within $K$ of one another. Then $\beta_{2}$ is $\left(\lambda_{2}(2 K+1)+\epsilon_{2}+K\right)$-tracked by $\beta_{1}$.

Proof. Since each vertex of $\beta_{1}$ is within $K$ of a vertex of $\beta_{2}$, we may define an integer function $a$ such that for each integer $i$ (in the domain of $\beta_{1}$ ), $\beta_{1}(i)$ is within $K$ of $\beta_{2}(a(i))$. We take $a(0)=0$ and if $\beta_{i}$ has $n_{i}$ edges then $a\left(n_{1}\right)=n_{2}$.

The first two inequalities follow from the definitions and the third follows from the first two.

1) $\quad \frac{|a(m+i)-a(m)|-\epsilon_{2}}{\lambda_{2}}-2 K \leq d\left(\beta_{2}(a(m+i)), \beta_{2}(a(m))\right)-2 K \leq$

$d\left(\beta_{1}(m+i), \beta_{1}(m)\right) \leq d\left(\beta_{2}(a(m+i)), \beta_{2}(a(m))\right)+2 K \leq|a(m+i)-a(m)|+2 K$

2)

$$
\frac{i-\epsilon_{1}}{\lambda_{1}} \leq d\left(\beta_{1}(m+i), \beta_{1}(m)\right) \leq i
$$

3)

$$
\frac{i-\epsilon_{1}}{\lambda_{1}}-2 K \leq|a(m+i)-a(m)| \leq
$$




$$
\lambda_{2}\left(d\left(\beta_{1}(m+i), \beta_{1}(m)\right)+2 K\right)+\epsilon_{2} \leq(i+2 K) \lambda_{2}+\epsilon_{2}
$$

The inequality $|a(i+1)-a(i)| \leq \lambda_{2}(2 K+1)+\epsilon_{2}$ implies if $k$ is between $a(i)$ and $a(i+1)$ for some $i$ then $\beta_{2}(k)$ is within $\lambda_{2}(2 K+1)+\epsilon_{2}+K$ of $\beta_{1}(i)$. In the case $\beta_{1}$ and $\beta_{2}$ are finite, the condition that terminal points are within $K$ of one another (so that $a\left(n_{1}\right)=n_{2}$ ) implies that every integer in the domain of $\beta_{2}$ is between $a(i)$ and $a(i+1)$ for some $i$ and this case is finished. If $\beta_{1}$ and $\beta_{2}$ are rays then $a(i)$ is non-negative and equation 3 ) (with $m=0$ ) implies $a(i)$ is arbitrarily large for large $i$ and again every integer in the domain of $\beta_{2}$ is between $a(i)$ and $a(i+1)$ for some $i$. If $\beta_{1}$ and $\beta_{2}$ are bi-infinite, then the $a(i)$ may be positive or negative and (again by 3$)$ ) for large $|i|,\left|a_{i}\right|$ is large, and $\lim _{i \rightarrow+\infty} a(i)= \pm \infty$ and $\lim _{i \rightarrow-\infty} a(i)= \pm \infty$. It remains to see $\lim _{i \rightarrow+\infty} a(i) \neq \lim _{i \rightarrow-\infty} a(i)$. Equality is impossible, since otherwise, for every large positive integer $i, a(-i)$ would be between $a(j)$ and $a(j+1)$ for some (depending on $i$ ) large positive integer $j$. But equation 3 ) implies $a(j)$ and $a(j+1)$ are relatively close and $a(-i)$ and $a(j)$ are far apart.

Proposition 4.2. Suppose $\beta$ is a quasi-geodesic edge path ray in $\Lambda$ and $\beta$ is tracked by a geodesic, then $\beta$ has bounded bracket number.

Proof. Assume that $\beta$ is a $(\lambda, \epsilon)$-quasi-geodesic. Suppose $\alpha$ is a geodesic such that each vertex of $\beta$ is within $L$ of a vertex of $\alpha$. For each integer $n \geq 0$, choose an integer $a(n)$ such that $d(\beta(n), \alpha(a(n))) \leq L$. We assume that $a(0)=0$.

The next two equations follow from the definitions and the third follows from the first two.

$$
\begin{gathered}
a(n)-2 L \leq d(\beta(n), \beta(0)) \leq a(n)+2 L \\
\frac{n-\epsilon}{\lambda} \leq d(\beta(n), \beta(0)) \leq n \\
\frac{n-\epsilon}{\lambda}-2 L \leq a(n) \leq n+2 L
\end{gathered}
$$

Claim 4.3. Suppose $K$ is an integer larger than $\lambda(4 L+1)+\epsilon$. Then for any integer $n, a(n+K)>a(n)$.

Proof. Note that if $m \geq \lambda(n+4 L)+\epsilon$ then $a(m)>n+2 L>a(n)$. So if $K>\lambda(4 L+1)+\epsilon$, and $a(n+K) \leq a(n)$, then there is a last integer $K_{1}>\lambda(4 L+1)+\epsilon$ such that $a\left(n+K_{1}\right) \leq a(n)$. Then (see figure 3 )

$$
a\left(n+K_{1}+1\right)>a(n) \geq a\left(n+K_{1}\right)
$$




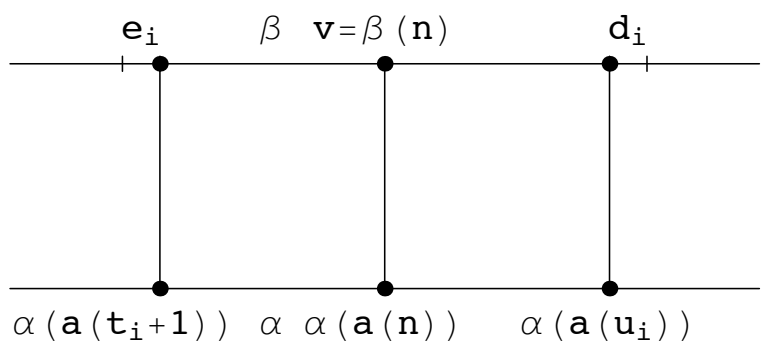

Figure 3

Since $d\left(\beta\left(n+K_{1}\right), \beta\left(n+K_{1}+1\right)\right)=1$ for all $n$, and $d(\beta(i), \alpha(a(i))) \leq L$ for all $i$, we have

$$
d\left(\alpha\left(a\left(n+K_{1}\right)\right), \alpha\left(a\left(n+K_{1}+1\right)\right)\right) \leq 2 L+1
$$

But as $\alpha(a(n))$ is between $\alpha\left(a\left(n+K_{1}\right)\right)$ and $\alpha\left(a\left(n+K_{1}+1\right)\right)$ on the geodesic $\alpha$,

$$
d\left(\alpha(a(n)), \alpha\left(a\left(n+K_{1}+1\right)\right)\right) \leq 2 L+1
$$

Then $d\left(\beta(n), \beta\left(n+K_{1}+1\right)\right) \leq 4 L+1$. But

$$
d\left(\beta(n), \beta\left(n+K_{1}+1\right)\right) \geq \frac{1}{\lambda}\left(K_{1}+1-\epsilon\right)>4 L+1
$$

the desired contradiction (so the claim is proved).

Now suppose $v \equiv \beta(n)$ is a vertex of $\beta$ with bracket number at least $2 \lambda(4 L+1)+2 \epsilon+K$. Then (by the pigeon hole principal) there are $K$ distinct walls, $Q_{1}, \ldots, Q_{K}$ such that for each $i \in\{1, \ldots, K\}$, there is an edge $e_{i}$ of $\beta$ preceding $v$ and an edge $d_{i}$ of $\beta$ following $v$ such that $e_{i}$ and $d_{i}$ belong to the wall $Q_{i}$, the subpath of $\beta$ between $e_{i}$ and $d_{i}$ does not cross $Q_{i}$, $e_{i}$ is not one of the $\lambda(4 L+1)+\epsilon$ edges of $\beta$ immediately preceding $v$ and $d_{i}$ is not one of the $\lambda(4 L+1)+\epsilon$ edges of $\beta$ immediately following $v$. I.e. $e_{i}=\beta\left(\left[t_{i}, t_{i}+1\right]\right)$ where $t_{i}+1 \leq n-\lambda(4 L+1)-\epsilon$ and $d_{i}=\beta\left(\left[u_{i}, u_{i}+1\right]\right)$ where $u_{i} \geq n+\lambda(4 L+1)+\epsilon$. (See figure 4.) 


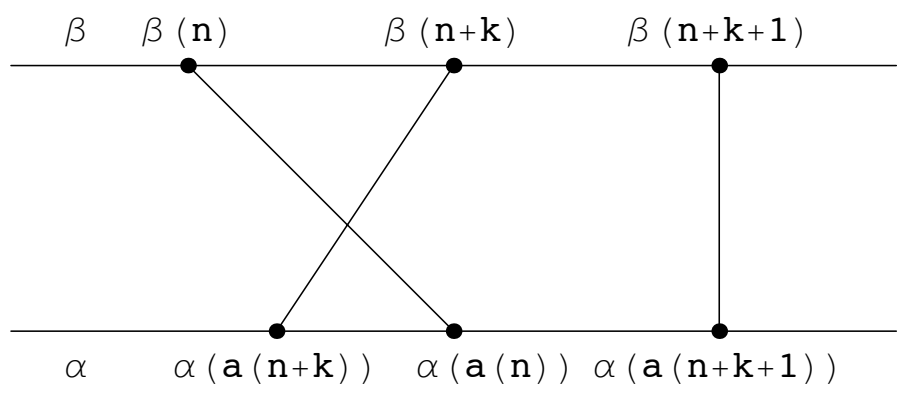

Figure 4

By claim 4.3, $a\left(t_{i}+1\right)<a(n)<a\left(u_{i}\right)$. Hence, by lemma 3.5, $\alpha(a(n))$ is within $2 L(2 P(1)+1)+P(1)$ of the wall $Q_{i}$. For $x$ a vertex of $\Lambda$, let $C(k)$ be the number of distinct walls that pass within $k$ of $x$. Note that $C$ is independent of vertex in $\Lambda$. Hence $K \leq C(2 L(2 P(1)+1)+P(1))$, bounding the bracket number of a vertex of $\beta$.

\section{Proof of Main Theorem}

In order to prove the main theorem, we need two results, one due to B. Brink and R. Howlett [BrH], and a second, due to R. P. Dilworth [Di].

Theorem 5.1. (Brink-Howlett) Suppose $(W, S)$ is a finitely generated Coxeter system, and $\Lambda(W, S)$ is the Cayley graph of $W$ with respect to $S$. There is a bound $F_{(W, S)}$ on the number of mutually crossing walls of $\Lambda$.

Dilworth's theorem requires several definitions. If $A$ is a partially ordered set (a set with reflexive, antisymmetric and transitive binary relation $\leq$ on $A$ ), then any two elements $x$ and $y$ are comparable if either $x \leq y$ or $y \leq x$. Otherwise they are in incomparable. A subset $C$ of $A$ is a chain when every pair of points in $C$ is a comparable pair. A subset $B$ of $A$ is called an anitchain when every pair of points in $B$ is an incomparable pair. The number of points in a maximal antichain is called the width of $A$.

Theorem 5.2. (Dilworth) If $A$ is a partially ordered set of width $w$, then $A$ can be partitioned into $w$ chains.

Suppose $x$ and $y$ are vertices of $\Lambda(W, S)$ and $\mathcal{W}_{(x, y)}$ is the set of walls that separate $x$ and $y$. We partially order $\mathcal{W}_{(x, y)}$ by saying $P \leq Q$ if either $P=Q$, or $P$ and $Q$ are parallel and $P$ separates $x$ from $Q$. Note that $P$ and $Q$ are parallel walls of $\mathcal{W}_{(x, y)}$, iff they are comparable. Hence $P$ and $Q$ are incomparable iff they cross. By proposition 5.1, the width of $\mathcal{W}_{(x, y)}$ is $F_{(W, S)}$. Applying Dilworth's theorem we have: 
Proposition 5.3. Suppose $(W, S)$ is a finitely generated Coxeter system, and $\Lambda(W, S)$ is the Cayley graph of $W$ with respect to $S$. For any vertices $x$ and $y$ of $\Lambda$ the walls separating $x$ and $y$ can be partitioned into at most $F_{(W, S)}$ chains (where any two walls in the same chain are parallel).

Say a path is geodesic with respect to a set of walls if the path crosses each wall of the set either 0 or 1 times. The following lemma is clear.

Lemma 5.4. Suppose $\alpha$ is an edge path in $\Lambda$ and $\alpha$ is geodesic with respect to the set of parallel walls $\mathcal{Q}$. If a subpath of $\alpha$ is replaced by a geodesic edge path, then the resulting edge path is geodesic with respect to $\mathcal{Q}$.

Theorem 5.5. Suppose $(W, S)$ is a finitely generated Coxeter system, $\alpha$ is a $(\lambda, \epsilon)$-quasi-geodesic edge path from a to $b$ in the Cayley graph $\Lambda(W, S)$. Then there is an integer $K$, depending only on $\Lambda, \lambda, \epsilon$ and the bracket number $B$ of $\alpha$, and $a \Lambda$-geodesic $\beta$ connecting $a$ and $b$ such that $\alpha$ is $K$-tracked by $\beta$.

Proof. The proof is a double induction argument. By proposition 5.3 , the walls separating $a$ and $b$ can be partitioned into at most $F$ sets $\mathcal{Q}_{1}, \ldots, \mathcal{Q}_{A}$, where two walls in the same set are parallel. The "outside" induction is on the number $A(\leq F)$ of sets of walls separating $a$ and $b$. The fact that $A$ is bounded by $F$ is critical to the argument that follows. Note that if $A=1$ then all walls separating $a$ and $b$ are parallel. In this case, the walls separating $a$ and $b$ are ordered as $Q_{1}, \ldots, Q_{m}$ where for $i<j<k$, $Q_{j}$ separates $Q_{i}$ from $Q_{k}$. Hence, there is a unique, geodesic edge path $\beta$ connecting $a$ and $b$, and $\beta$ crosses $Q_{1}$, then $Q_{2}, \ldots$ By proposition 3.3 , the path $\alpha$ is approximated by a path $\alpha^{\prime}$, such that each vertex of $\alpha^{\prime}$ is on a geodesic connecting $a$ and $b$. The path $\alpha^{\prime}$ only crosses the walls separating $a$ and $b$ (see lemma 3.4) and, in this case, is geodesic, modulo backtracking. Eliminating backtracking on $\alpha^{\prime}$ produces $\beta$. Each vertex of $\alpha^{\prime}$ is a vertex of $\beta$ and the basis case is complete.

Assume the statement of the theorem is true if $A$, the number of sets of walls separating $a$ and $b$, is less than or equal to $M-1$. Suppose there are $M$ sets of walls $\left(\mathcal{Q}_{1}, \ldots, \mathcal{Q}_{M}\right)$ separating $a$ and $b$. By proposition 3.3 we may assume every vertex of $\alpha$ is on a geodesic connecting $a$ and $b$, so that $\alpha$ only crosses walls separating $a$ and $b$ and $\alpha$ crosses each such wall an odd number of times. The second induction is on $N(\leq M)$, the number of sets of walls, $\mathcal{Q}_{i}$, such that $\alpha$ is not geodesic with respect to $\mathcal{Q}_{i}$. If $N=0$, then $\alpha$ is geodesic. Assume the statement of the theorem is true for $N=K-1$ (when the number of sets of walls separating $a$ and $b$ is $\leq M$ ). Assume the $\mathcal{Q}_{i}$ are arranged so that $\alpha$ is geodesic with respect to $\mathcal{Q}_{i}$ for $K+1 \leq i \leq M$. Write $\alpha$ as $\left(e_{1}, \ldots, e_{n}\right)$ with consecutive vertices $a \equiv a_{1}, \ldots, a_{n} \equiv b$. Let $i$ be the first integer such that $e_{i}$ is an edge of a wall of $\mathcal{Q}_{K}$ and for some $j>i, e_{j}$ and $e_{i}$ are in the same wall $Q$. Now assume $j$ is the largest integer such that $e_{j} \in Q$. Since $\alpha$ crosses $Q$ an odd number of times, the path $\alpha_{i, j} \equiv\left(e_{i}, \ldots, e_{j-1}\right)$ (from $a_{i}$ to $a_{j}$ ) crosses $Q$ an even number of times. A geodesic $\beta_{i, j}$ connecting $a_{i}$ to $a_{j}$ does not cross $Q$. Since all walls of $\mathcal{Q}_{K}$ are 
parallel to one another, $\beta_{i, j}$ does not cross a wall of $\mathcal{Q}_{K}$. Hence $a_{i}$ and $a_{j}$ are not separated by a wall of $\mathcal{Q}_{K}$. By proposition 3.3 , $\alpha_{i, j}$ is close to $\alpha_{i, j}^{\prime}$ a quasi-geodesic edge path connecting $a_{i}$ to $a_{j}$, such that each vertex of $\alpha_{i, j}^{\prime}$ is on a geodesic connecting $a_{i}$ to $a_{j}$. By lemma 3.4, each wall separating $a_{i}$ and $a_{j}$ also separates $a$ and $b$, and the number of sets of walls separating $a_{i}$ and $a_{j}$ is less than $M$. By (outside) induction, there is a geodesic $\beta_{i, j}$ connecting $a_{i}$ and $a_{j}$ which tracks $\alpha_{i, j}^{\prime}$ and therefore tracks $\alpha_{i, j}$. Replace $\alpha_{i, j}$ by $\beta_{i, j}$. The resulting path, $\alpha_{1}$ crosses $Q$ exactly once at $e_{j}$. The walls of $\mathcal{Q}_{K}$ are ordered as $Q_{1}, Q_{2}, \ldots$ so that if $i<j$, then $Q_{i}$ separates $a$ from $Q_{j}$, and $Q_{j}$ separates $Q_{i}$ from $b$. A wall of $\mathcal{Q}_{K}$ preceding $Q$ in this ordering is not crossed by $\alpha_{1}$ after $e_{j}$. Hence if $\mathcal{Q} \subset \mathcal{Q}_{K}$ is the set of walls of $\mathcal{Q}_{K}$ preceding $Q$ and including $Q$, then $\alpha_{1}$ is geodesic with respect to $\mathcal{Q}$ and (by lemma 5.4), $\alpha_{1}$ is geodesic with respect to each set $\mathcal{Q}_{i}$ for $i>K$. Suppose $e_{k}$ is the first edge of $\alpha_{1}$ such that $e_{k}$ is an edge of a wall $Q$ of $\mathcal{Q}_{K}$, and for some $l>k, e_{l} \in Q$. Then $e_{k}$ follows $e_{j}$ on $\alpha_{1}$, and if we assume $e_{l}$ is the last edge of $\alpha_{1}$ in $Q$, then as above $\left(e_{k}, \ldots, e_{l-1}\right)$ can be replaced by a geodesic close to $\left(e_{k}, \ldots, e_{l-1}\right)$. Continuing, the resulting path is geodesic with respect to $\mathcal{Q}_{K}$ and by induction, the theorem follows. Note that the bound $F$ for $\Lambda$ (on the number of sets of parallel walls are necessary to partition the set of walls separating two points $a$ and $b$ of $\Lambda$ ), limits the total number of times the induction steps are carried out to arrive at a geodesic.

\section{Consequences of the Main Theorem}

Corollary 6.1. Suppose $(W, S)$ is a finitely generated Coxeter system, and $\Lambda(W, S)$ is the Cayley graph of $W$ with respect to $S$. Any infinite or biinfinite $(\lambda, \epsilon)$-quasi-geodesic edge path $\alpha$ with bounded bracket number $B$ is $K^{\prime}$-tracked by an edge path geodesic where $K^{\prime}$ is a constant only depending on $\lambda, \epsilon, B$ and $S$.

Proof. The proof is a standard local finiteness argument in both the infinite and bi-infinite case. We give the bi-infinite case. Write $\alpha$ as the edge path $\left(\ldots, e_{-1}, e_{0}, e_{1}, \ldots\right)$ in $\Lambda$. Let $v_{i}$ be the initial point of $e_{i}$. By theorem 5.5 , there is a $\Lambda$-geodesic $\beta_{n}$ which $K$-tracks $\alpha_{n} \equiv\left(e_{-n}, \ldots, e_{n}\right)$. Note that every vertex of $\beta_{n}$ is within $2 K$ of a vertex of $\alpha$. For each positive integer $n$, some vertex $x_{n}$ of $\alpha_{n}$ is within $K$ of $v_{0}$. Hence there is an infinite number of $x_{n}$ that are equal. Of this infinite subcollection of $x_{n}$, infinitely many have the same pair of edges one preceding and one following $x_{n}$ on $\beta_{n}$, of this infinite collection of $x_{n}$ there is an infinite subcollection that have the same four edges - the two preceding and the two following $x_{n}$ being exactly the same. Continuing, we have a bi-infinite geodesic $\beta$ and each vertex of $\beta$ is within $2 K$ of a vertex of $\alpha$. As $\alpha$ is a $(\lambda, \epsilon)$-quasi-geodesic, lemma 4.1 implies each point of $\alpha$ is within $\lambda(4 K+1)+\epsilon+2 K$ of $\beta$.

The next result follows directly from proposition 4.2 and corollary 6.1. 
Corollary 6.2. Suppose $(W, S)$ is a finitely generated Coxeter system, and $\Lambda(W, S)$ is the Cayley graph of $W$ with respect to $S$. Then a quasi-geodesic edge path ray in $\Lambda$ is tracked by a geodesic iff it has bounded bracket number.

A metric space $(X, d)$ is a called a geodesic metric space if every pair of points are joined by a geodesic. It is proper if for any $x \in X$, the ball of radius $r$ about $X$ is compact for all positive numbers $r$. A group $W$ acts geometrically on a space if the action is properly discontinuous, co-compact and by isometries.

Let $(X, d)$ be a proper complete geodesic metric space. If $\triangle a b c$ is a geodesic triangle in $X$, then we consider $\Delta \bar{a} \bar{b} \bar{c}$ in $\mathbb{E}^{2}$, a triangle with the same side lengths, and call this a comparison triangle. Then we say $X$ satisfies the $C A T(0)$ inequality if given $\triangle a b c$ in $X$, then for any comparison triangle and any two points $p, q$ on $\triangle a b c$, the corresponding points $\bar{p}, \bar{q}$ on the comparison triangle satisfy

$$
d(p, q) \leq d(\bar{p}, \bar{q})
$$

If $(X, d)$ is a $C A T(0)$ space, then the following basic properties hold:

(1) The distance function $d: X \times X \rightarrow \mathbb{R}$ is convex.

(2) $X$ has unique geodesic segments between points.

(3) $X$ is contractible.

For details, see $[\mathrm{BH}]$.

Suppose $(W, S)$ is a finitely generated Coxeter system, $\Lambda(W, S)$ is the Cayley graph of $W$ with respect to $S$ and $W$ acts geometrically on a CAT $(0)$ space $X$. Define $\Lambda_{x} \subset X$ to have as vertices, the orbit $W x$, and CAT(0) geodesic edge connecting $w_{1} x$ and $w_{2} x$ (for $w_{i} \in W$ ) when there is $s \in S$ such that $w_{1} s=w_{2}$. There is a proper $W$-equivariant map $P_{x}: \Lambda \rightarrow \Lambda_{x}$ so that $P_{x}$ maps the identity vertex of $\Lambda$ to $x$.

Intuitively, the next result says that when a Coxeter group acts geometrically on a $\mathrm{CAT}(0)$ space, $\mathrm{CAT}(0)$ geodesics are tracked by Cayley graph geodesics. This result generalizes the right angled version of the same result in MRT.

Corollary 6.3. Suppose $(W, S)$ is a finitely generated Coxeter system, and $\Lambda(W, S)$ is the Cayley graph of $W$ with respect to $S$ and $W$ acts geometrically on the proper $C A T(0)$ space $X$. If $x$ is a point of $X$ not fixed by any element of $W$, and $\Lambda_{x}$ is the copy of $\Lambda$ at $x$, then any $C A T(0)$ geodesic ray in $X$ is tracked by a Cayley graph geodesic in $\Lambda_{x}$.

Proof. For a given CAT(0) geodesic $\alpha$ we find a Cayley graph geodesic $\beta$ such that $P_{x}(\beta)$ tracks $\alpha$. It suffices to find $\lambda, \epsilon, K$ and $B$ such that any (finite) $\mathrm{CAT}(0)$ geodesic $\alpha$ is $K$-tracked by a Cayley $(\lambda, \epsilon)$-quasi-geodesic with bracket number $\leq B$. Since $W$ acts co-compactly on $X$, there is an integer $K_{1}$ such that every point of $X$ is within $K_{1}$ of the orbit $W x$. For each integer $0,1, \ldots, N$ such that $N$ is less that or equal to the length of $\alpha$, choose a point $v_{i} x$ of $W x$ within $K_{1}$ of $\alpha(i)$. Let $\beta_{i}$ be a $\Lambda$-geodesic 
connecting $v_{i}$ to $v_{i+1}$ and $\beta$ be the $\Lambda$-edge path $\left(\beta_{0}, \beta_{1}, \ldots\right)$. Since the map $P_{x}: \Lambda \rightarrow \Lambda_{x}$ is quasi-isometric, there are numbers $\lambda$ and $\epsilon$ such that any such $\beta$ is a $(\lambda, \epsilon)$-quasi-geodesic in $\Lambda$ and numbers $D_{\Lambda}$ and $D_{X}$ such that the length of any $\beta_{i}$ is less than or equal to $D_{\Lambda}$ (in $\Lambda$ ) and every point of such a $P_{x}\left(\beta_{i}\right)$ is within $D_{X}$ of $\alpha(i)$ (in $X$ ). Certainly every point of $\alpha$ is within $K \equiv K_{1}+1$ of $P_{x}(\beta)$.

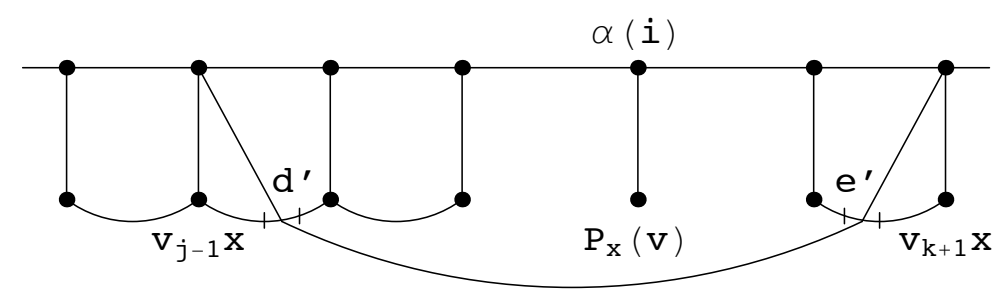

Figure 5

Hence it suffices to bound the bracket number of such a $\beta$. If $v$ is a vertex of $\beta_{i}$ and $e$ and $d$ are edges of $\beta$ preceding and following $v$ respectively such that $e$ and $d$ belong to the same wall $Q$ of $\Lambda$, then $e$ is an edge of $\beta_{j}$ and $d$ is an edge of $\beta_{k}$ where $j \leq i \leq k$. The mid-points $e^{\prime}$ of $P_{x}(e)$ and $d^{\prime}$ of $P_{x}(d)$ are fixed (in $\Lambda_{x}$ and $X$ ) by the reflection $r_{Q} \in W$ for the wall $Q$ (as are the mid-points of $e$ and $d$ in $\Lambda$ ). Hence the geodesic in $X$ connecting $d^{\prime}$ and $e^{\prime}$ is fixed by $r_{Q}$. Now, $d^{\prime}$ (respectively $e^{\prime}$ ) is within $D_{X}$ of $\alpha(j-1)$ (respectively $\alpha(k+1)$ ) and $P_{x}(v)$ is within $D_{X}$ of $\alpha(i)$. By the $\operatorname{CAT}(0)$ inequality for quadrilaterals (in particular for the quadrilateral determined by $d^{\prime}, e^{\prime}, \alpha(j-1)$, and $\left.\alpha(k+1)\right) \alpha(i)$ is within $D_{X}$ of a point of the $X$ geodesic connecting $d^{\prime}$ to $e^{\prime}$ and hence $\alpha(i)$ is within $D_{X}$ of a fixed point of $r_{Q}$. (See figure 5.)

Since the action of $W$ on $X$ is properly discontinuous, there is a bound $B$ on the number of reflections $r_{Q}$ such that $r_{Q}$ does not take the ball of radius $D_{X}$ centered at $v(i) \in X$ (equivalently centered at any $x \in X$ ) off of itself. Hence there cannot be more than $B$ walls bracketing the vertex $v$ of $\beta$.

Remark 6.4. Note that the above proof is valid even when $W$ does not act co-compactly on the $C A T(0)$ space $X$, as long as the $C A T(0)$ geodesic remains a bounded distance from $\Lambda_{x}$ for some $x$.

The following result answers a question posed by K. Ruane.

Corollary 6.5. Suppose $(W, S)$ is a finitely generated Coxeter group acting geometrically on the CAT(0) space $X$. For $x \in X$ let $\Lambda_{x}$ be the copy of the Cayley graph of $(W, S)$ in $X$, (with $W$-equivariant map $P_{x}: \Lambda(W, S) \rightarrow \Lambda_{x}$ ). Then for each subset $A \subset S$, the subgroup $\langle A\rangle$ is quasi-convex in $X$. (I.e. $P_{x}(\langle A\rangle)$ is quasi-convex in $X$.)

Proof. Let $K$ be the tracking constant from corollary 6.3. Suppose $a_{1}, a_{2} \in$ $A$ and $\alpha$ is a $\operatorname{CAT}(0)$ geodesic in $X$ from $P_{x}\left(a_{1}\right)$ to $P_{x}\left(a_{2}\right)$. Let $\beta$ be a $\Lambda_{x}$, 
edge path geodesic which $K$-tracks $\alpha$. I.e. there is a $\Lambda(W, S)$ geodesic $\beta^{\prime}$, from $a_{1}$ to $a_{2}$ such that $P_{x}\left(\beta^{\prime}\right)=\beta$. Since $a_{i} \in A$, the edge labels of $\beta^{\prime}$ are all in $A$. This means all vertices of $\beta^{\prime}$ are in $\langle A\rangle$, and so the image of $\alpha$ is within $K$ of $P_{x}(\langle A\rangle)$.

The next result says that elements of infinite order in a Coxeter group are tracked by geodesics in the standard Cayley graph.

Corollary 6.6. Suppose $(W, S)$ is a finitely generated Coxeter system and $g \in W$ is an element of infinite order. Then in the Cayley graph $\Lambda(W, S)$ the elements $\left\{\ldots, g^{-2}, g^{-1}, 1, g, g^{2}, \ldots\right\}$ are tracked by a Cayley graph geodesic.

Proof. By G. Moussong [Mo, all finitely generated Coxeter groups are CAT(0). Let $X$ be any CAT(0) space such that $W$ acts geometrically on $X$. The min set of $g$ contains a geodesic line $l$ that is invariant under the action of $g$. Let $x$ be any point in $X$ and $\Lambda_{x}$ the copy of $\Lambda(W, S)$ in $X$ at $x$. Let $\alpha$ be an $S$-geodesic for $g$. Observe that the edge path line $l_{g}$ in $\Lambda_{x}$ determined by positive and negative iterates of $\alpha$ at $x$ is a bounded distance from $l$. The proof of corollary 6.3 shows that $l_{g}$ is a quasi-geodesic with bounded bracket number and so by corollary 6.1 is tracked by a Cayley graph geodesic.

One of the fundamental asymptotic results for word hyperbolic groups is that 1-ended word hyperbolic groups have locally connected boundary. This result follows from a long program of results by several authors, notably B. Bowditch, and concluded by G. Swarup [S]. To give a feeling for the reach of our results, we outline an elementary proof of this fact for Coxeter groups.

Corollary 6.7. If $W$ is a 1-ended word hyperbolic Coxeter group then the boundary of $W$ is locally connected.

Proof. We use an elementary form of a construction of a "filter" in [MRT] (where a partial classification of right angled Coxeter groups with locally connected boundaries is produced). Suppose $W$ acts geometrically on the CAT(0) space $X$, with base point $x$. Let $\Lambda_{x}$ be the copy of the Cayley graph of $(W, S)$ at $x$ in $X$ with proper $W$-equivariant map $P_{x}: \Lambda(W, S) \rightarrow$ $\Lambda_{x}$. Suppose $r$ and $s$ are "close" geodesic rays in $X$, with $r(0)=s(0)=$ $x$. Choose $\Lambda$ (edge path) geodesics $r^{\prime}$ and $s^{\prime}$ at $*$ (the identity vertex of $\Lambda(W, S))$, such that $P_{x}\left(r^{\prime}\right)$ and $P_{x}\left(s^{\prime}\right) K$-track $r$ and $s$ respectively. Since $r$ and $s$ are close in $\partial X$, we may assume that $r^{\prime}$ and $s^{\prime}$ have long initial segments with "close" terminal points. For simplicity we assume these initial segments agree. If $y$ is the last vertex of this common initial segment, say the edge of $r^{\prime}$ following $y$ has label $a_{1}$ and the edge of $s^{\prime}$ following $y$ has lablel $b_{1}$. The presentation diagram $\Gamma(W, S)$ of $(W, S)$ has vertex set $S$ and an edge labeled $m(i, j)$ between distinct vertices $s_{i}, s_{j}$ if $m(i, j) \neq \infty$. Since $W$ is 1-ended no subset $A$ of $S$ with $\langle A\rangle$ a finite group separates $\Gamma$ (see corollary 16 of [MT]). The set $B$ of $S$-elements that label edges at $y$ with end points closer to $*$ than $y$ is to $*$ generates a finite subgroup of $W$. The set of vertices of $\Gamma$ corresponding to $B$ does not separate $\Gamma$ and $B$ does not 
contain $a_{1}$ or $b_{1}$. Hence there is an edge path in $\Gamma$ from $a_{1}$ to $b_{1}$ avoiding $B$. Let the consecutive vertices of this path be $a_{1}=v_{1}, v_{2}, \ldots, v_{n}=b_{1}$. If $q(i, i+1)$ is the (finite) order of $v_{i} v_{i+1}$ then the relation $\left(v_{i}, v_{i+1}\right)^{q(i, i+1)}$ determines a loop at $y \in \Lambda$ such that the two half loops at $y$ making up this loop extend the Cayley geodesic from $*$ to $y$. Consider the subgraph $F_{1}$ of $\Lambda$ determined by the edge paths $r^{\prime}, s^{\prime}$ and the edge loops for each $v_{i} v_{i+1}$. Each $v_{i}$ determines an edge of $F_{1}$ (with label $v_{i}$ ) beginning at $y$. At the end point of this edge there are two edges of $F_{1}$ that extend a Cayley geodesic from $*$ to $y$. Build a set of loops as with $a_{1}$ and $b_{1}$ for each of these pairs of edges. Then $F_{2}$ is $F_{1}$ union all new loops. Continuing we build a 1-ended subgraph $F=\cup_{i=1}^{\infty} F_{i}$ of $\Lambda$ such that for each vertex $v$ of $F$, not on the common overlap of $r^{\prime}$ and $s^{\prime}$, there is a Cayley geodesic from $*$ to $v$ in $F$ which passes through $y$. We claim that $L$, the limit set of $P_{x}(F)$ is a "small" connected set containing $r$ and $s$ (and so $\partial X$ is locally connected). Certainly, $r$ and $s$ are in $L$. Since $F$ is 1-ended and $P_{x}$ is proper, $L$ is connected. If $v$ is a vertex of $F$, then there is a Cayley geodesic $\alpha_{v}$ from $*$ to $v$ (which passes through $y$ for all but finitely many $v$ ). If $z \in L$ then let $z_{1}, z_{2}, \ldots$ be a sequence of vertices of $F$ such that $P_{x}\left(z_{i}\right)$ converges to $z$. The CAT $(0)$ geodesic from $x$ to $P_{x}\left(z_{i}\right)$ is $K$ - tracked by a Cayley geodesic $\beta_{i}$ in $\Lambda_{x}$. As $W$ is word hyperbolic the Cayley geodesics $P_{x}\left(\alpha_{v_{i}}\right)$ and $\beta_{i}$ (with the same end points) must $\delta$-fellow travel (for a fixed constant $\delta$ ). In particular each $\beta_{i}$ must pass "close" to $P_{x}(y)$ and so $z$ is close to both $r$ and $s$ in $\partial X \equiv \partial W$.

\section{REFERENCES}

[BH] M.R. Bridson, A. Haefliger. Metric Spaces of Non-positive Curvature (Grundl. Math. Wiss., Vol. 319, Springer, Berlin 1999).

[BrH] B. Brink, R.B. Howlett. A finiteness property and an automatic structure for Coxeter groups. Math. Ann. 296(1) (1993), 179-190.

[D] M.W. Davis, The geometry and topology of Coxeter groups. London Mathematical Society Monographs Series Vol. 32, Princeton University Press, Princeton, NJ, 2008.

[Di] R.P. Dilworth. A Decomposition Theorem for Partially Ordered Sets. Ann. of Math. 51 (1950), 161-166.

[MRT] M. Mihalik, K. Ruane, S. Tschantz. Local connectivity of right angled Coxeter group boundaries. J. Group Theory 10 (2007), 531-560.

[MT] M. Mihalik, S. Tschantz. Visual decompositions of Coxeter grouip. Groups Geom. Dyn. 3 (2009), 173-198.

[Mo] G. Moussong. Hyperbolic Coxeter groups. PhD. thesis. Ohio State University (1988).

[Sh] H. Short (ed.) Notes on word hyperbolic groups. In Group Theory from a Geometrical Viewpoint (E. Ghys, A. Haefliger and A. Verjovsky ed.) World Scientific 1990, 3-64.

[S] G. Swarup. On the cut point conjecture. Electron. Res. Announc. Amer. Math. Soc. 2 (1996).

Department of Mathematics, Vanderbilt University, Nashville, TN 32340

E-mail address: michael.1.mihalik@vanderbilt.edu

E-mail address: steven.tschantz@vanderbilt.edu 